\title{
PROYECTO DE INNOVACIÓN: IMPLEMENTACIÓN DE UN SISTEMA DE CAPACITACION VIRTUAL
}

\author{
Eduardo Méndez*
}

\begin{abstract}
El artículo presenta la implementación y el desarrollo de un sistema de capacitación corporativa a distancia a través de ambientes virtuales. Se trata de una propuesta de innovación inserta en un importante organismo estatal uruguayo.

Ésta implica un significativo cambio de estrategias en la capacitación institucional determinando importantes desafíos a nivel de cultura y cambio organizacional.

Se describe la realidad actual de la organización, lo cual permite la comprensión necesaria del ámbito para el cual se diseñó la innovación. En este marco, se fundamenta la relevancia de las acciones propuestas y la metodología empleada.

El marco conceptual se construye a partir de diversas áreas disciplinares recibiendo aportes desde la concepción de innovación, la didáctica, el aprendizaje, la educación a distancia y la tecnología educativa.

En la metodología se desarrolla el componente de innovación, planteando un segundo componente de investigación a nivel de diseño; éste será incorporado como un factor de evaluación de la innovación en una etapa posterior.

Finalmente se describe el estado actual del proyecto, incluyendo los productos ya desarrollados y entregados, así como los pasos a seguir en las siguientes etapas.

\section{1- Introducción}

"... no dejar que el pasado se plante en nuestro camino hacia el futuro, porque el futuro va a ser diferente y tenemos que desaprender la manera como hemos aprendido y actuado en el pasado, para poder aprender y actuar en el futuro". La expresión de Toffler es una buena representación de un proceso vinculado a la innovación. Ésta transita por el cambio, por la mejora, sin embargo no cualquier cambio significa innovar. La innovación lleva implícita una ruptura, ruptura con las formas tradicionales de hacer, con la manera "convencional", con lo establecido, ruptura que necesariamente debe afectar las bases de lo innovado, impactando en las realidades y representaciones de las instituciones, de los hechos y de los actores. En este camino inexorablemente se transita por "desaprendizajes" y nuevos aprendizajes.

Este artículo nos presentará una innovación de corte institucional realizada en un organismo estatal uruguayo, cuya gestión se ha visto complejizada por los actuales escenarios de incertidumbre y dinamismo y el permanente surgimiento e incorporación de nuevas tecnologías de información y comunicación.

Asumiendo el reto de mejorar la capacitación a nivel corporativo se gestó la formulación de un proyecto de innovación, proyecto que ya está en etapa de realización y da cuenta de la transferencia de los aportes del ámbito académico a una realidad organizacional particular.

La propuesta expone el desarrollo de la modalidad de capacitación a distancia a través de ambientes virtuales. Ésta se formula desde la unidad que tiene por cometido las competencias del área, contando con la posibilidad de presentar e implementar propuestas y la ventaja de que los niveles de decisión están orientados hacia la mejora continua.
\end{abstract}


La proposición implicó un importante cambio de estrategias en la capacitación corporativa y ha presentado enormes desafíos, sobre todo a nivel de cultura organizacional. Hacer las cosas de otro modo implica, ineludiblemente, el reto de la superación de las resistencias que se generan. No menos importante para este logro es señalar que la innovación se gesta en un contexto de fuerte restricción de gastos, lo cual impacta directamente en la disponibilidad de recursos.

\section{2-Propósito y ámbito de la innovación}

Se estableció como objetivo del proyecto la concepción, el diseño y la implementación de un sistema de capacitación en competencias laborales, con procesos de gestión y evaluación a través de medios alternativos, con énfasis en la utilización de aplicativos en ambiente Intranet.

Para aclarar su alcance es necesario definir el término "capacitación" desde la acepción que el mismo tiene para la institución en particular. Éste coincide con el significado planteado por Blake "como la parte de la educación de los adultos que se vincula con su vida de trabajo" (1997:21). En otras palabras, el ambiente de enseñanzas y aprendizajes provistos por la organización que proporcionan al trabajador las competencias necesarias para el efectivo desempeño de su labor.

Expresado esto, es importante señalar además que, toda experiencia innovadora debe construirse esencialmente desde el ámbito que le da significación a las prácticas. El desafío está entonces en comprender y construir el trabajo a partir de ese lugar, con el fin de otorgarle su verdadero sentido, eficacia y permanencia.

Describiendo escuetamente esa realidad diremos que la organización en la cual se desarrolla la propuesta se encuentra desde el último lustro en un profundo proceso de cambio. Éste es determinado por múltiples factores, entre ellos las políticas públicas, las transformaciones propias de gestión (orientada hacia procesos de reingeniería y mejora continua) y el establecimiento de directrices estratégicas centradas en las necesidades de sus clientes.

Caracterizando este proceso podemos señalar que el mismo se traduce en:

- Importantes cambios de normativa y procesos internos que atraviesan todas las áreas de negocios de la Institución.

- Alta informatización organizacional (en equipamiento y procesos).

- Descentralización.

- Profesionalización de los cuadros de Recursos Humanos.

- Reducción permanente de la plantilla de personal.

En este contexto se vuelve vital la necesidad -en tiempo, forma y calidad- de capacitar, informar, actualizar y motivar al personal, elementos claves para alcanzar y sustentar los desafíos planteados por el cambio.

En este escenario, el área de Recursos Humanos determina las competencias funcionales, las cuales son establecidas de acuerdo al perfil específico de cada puesto ocupacional. En otros términos, cada puesto requiere determinados conocimientos, aptitudes y actitudes. La capacitación institucional ha sido y es el pilar en la generación de las mismas, compensando las brechas que se presentan entre el perfil del individuo y del puesto. Este proceso es permanente y se provee a través de una Unidad especializada la cual desarrolla sus acciones mediante cursos presenciales, en su mayoría realizados por Instructores Internos. 


\section{Fundamentación}

Los cursos en modalidad presencial se ejecutan con un alto grado de eficacia; sin embargo, resulta esencial considerar los siguientes aspectos:

- la dispersión que presentan los funcionarios debido a la propia amplitud (organizacional y geográfica) de la Institución,

- la rapidez con la cual se suceden los cambios y el grado de especialización que estos van generando,

- la preocupación por disminuir el impacto en la producción operativa en tanto se ejecuta la capacitación (sobre todo en consideración de la reducción de personal y su disponibilidad de tiempo),

- la necesidad de lograr un aumento en la calidad de la producción académica,

- la presencia de nuevas tecnologías informáticas y en comunicación,

- las actuales modalidades de capacitación que se van imponiendo por su eficiencia y posibilidades.

Este contexto hace necesario orientar la capacitación y formación del personal hacia modalidades alternativas, en especial aquellas que presenten un alto grado de sofisticación tecnológica que apoyen la mejora y el acrecentamiento de las estrategias de aprendizaje autónomo en los estudiantes.

Es necesario poner énfasis que esta innovación no es concebida simplemente como la generación de un ambiente de entrega de información o de capacitación puntual, sino desde un objetivo mucho más amplio: el del un proceso formativo en donde conocimiento y desarrollo son las dos caras de una misma moneda. Este proceso es altamente complejo e involucra lo social, lo cultural y lo interpersonal. Su riqueza radica justamente en su complejidad y en la enorme variedad de factores implicados. Entre ellos podemos hallar la comunicación de los conocimientos, el desarrollo personal de quienes están involucrados, la facilitación y el establecimiento de las relaciones emocionales y sociales, las características de los conocimientos, la activación del proceso intelectual y cognoscitivo: en suma, los factores involucrados en la creación del saber hacer y el saber ser del aprendiz.

El modelo educativo basado en la modalidad virtual deberá permitir formas de interacción en la tríada saber-enseñante-aprendiz que promuevan la consecución de este objetivo y que a su vez contemplen la complejidad expresada. Pensamos que esta modalidad le proporcionará uno de los más ricos aportes, al permitir combinar propuestas presenciales con nuevos modos de desarrollar el acto educativo, a través de la incorporación de nuevas tecnologías.

\section{Marco conceptual}

La formulación de los conceptos claves que orientaron esta propuesta de cambio contextualizada en un ambiente laboral y modalidad no presencial, que tiene implícito además un intenso impacto en la dirección estratégica de capacitación, requirió del aporte de varias fuentes.

Este proceso se construye entonces considerando los aportes disciplinares provistos desde la concepción de innovación, la didáctica, el aprendizaje, la educación a distancia y finalmente la tecnología educativa.

La concepción de innovación permite pensar y construir la propuesta en términos implementables. Un trabajo orientado hacia la innovación requiere comprender su naturaleza, cuáles son las condiciones en que se produce y desarrolla, sus características y qué mecanismos de la estructura institucional incidirán fomentando o recortando el emprendimiento.

A su vez, esta experiencia implica un cambio profundo del escenario en donde tienen lugar los procesos de enseñanza. Debemos entonces considerar que las relaciones entre los componentes enseñante - aprendiz y saber son diferentes, debiéndose pensar desde la didáctica nuevas y eficaces formas de presentación de los saberes y de conducción del proceso de enseñanza. 


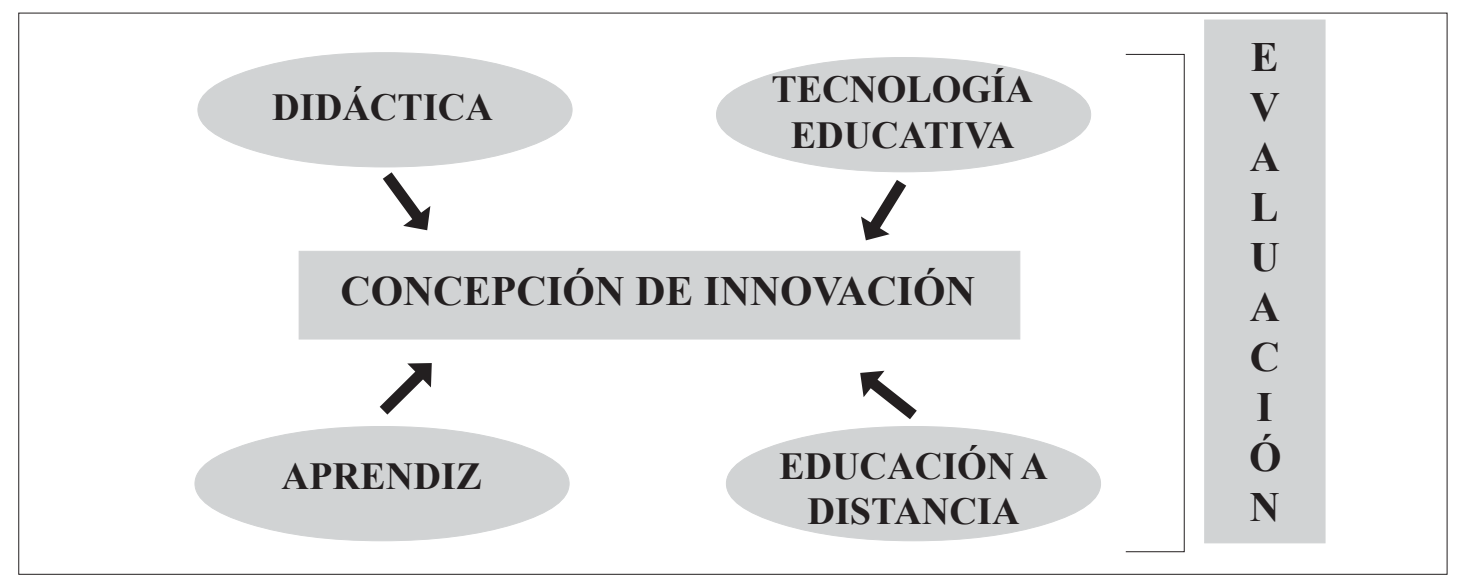

Esquema: Construcción del marco conceptual

Por otro lado, el propósito del trabajo (establecer un sistema no presencial de capacitación en competencias laborales) no puede obviar la necesidad de la comprensión de cuáles son las concepciones de aprendizaje, factores que inciden y principales aspectos cognitivos involucrados en esta modalidad.

En el marco de la capacitación virtual, docente y estudiante están separados por la distancia o por distancia y tiempo, y dependerán de la tecnología como mediador en el proceso de la comunicación. Requerimos entonces aportes desde la concepción de educación a distancia que nos señalen los factores involucrados en la relación de enseñanzas y aprendizajes planeados en lugares y tiempos diferentes al de los docentes y de la tecnología educativa que nos orientará conceptualmente respecto de los medios y herramientas destinadas a la creación de materiales

Finalmente dejaremos expresado, a nivel de propuesta, un componente adicional de investigación, el cual se orienta a cumplir la función de evaluación de la propuesta.

\section{Aportes disciplinares}

\section{Desde la concepción de innovación}

Como se expresó, plantearnos un trabajo orientado hacia la innovación requiere comprender su naturaleza: qué se entiende por tal, cuáles son las condiciones en que se produce, qué características tiene este proceso y qué mecanismos de la estructura institucional inciden favoreciendo o inhibiendo el emprendimiento.

Estas concepciones son abordadas asumiendo la orientación planteada por Aguerrondo, quien considera a la innovación como "todo intento de ruptura del equilibrio dado por el funcionamiento rutinario..." (1996: 20-21), asimismo señala que para que una experiencia sea considerada innovadora se requiere afectar los ejes fundantes de la estructura, de lo contrario, "no se puede hablar en sentido propio de innovación..." (Aguerrondo, op cit).

En relación a lo expresado, la propuesta implica un sustancial cambio del escenario donde tienen lugar los procesos de enseñanza y aprendizaje con una importante afectación de la dimensión técnico pedagógica, así como también cambios a nivel de organización incidiendo fuertemente en la dimensión político - ideológica.

Una idea de la afectación de los ejes fundantes nos la proporciona la enunciación de las expectativas sobre los beneficios previstos, entre otros: 
- Que los productos se adapten a los distintos ritmos de aprendizaje y horarios de trabajo permitiendo al usuario controlar su avance y organizar su tiempo.

- Las distintas posibilidades brindadas desde el ambiente virtual deben permitir el logro equivalente o producir una mejora en aspectos tales como: la eficacia en el feedback hacia el alumno, el proceso de actualización de contenidos, la disponibilidad del curso, la optimización de la transmisión del conocimiento del personal referente y la simplificación de la gestión administrativa.

- Que los participantes reciban la misma información y una idéntica presentación de contenidos, la calidad y el enfoque con el que fue concebido el material se deben mantener constantes.

- El usuario debe poder acceder desde su puesto de trabajo a la sesión de capacitación evitando combinaciones estrictas de fechas.

- Proporcionar productos que permitan evaluar el nivel de aprendizaje, la actividad de los usuarios (horas de estudios dedicadas) y su tracking (qué unidades programáticas vio).

- Producir una disminución de impacto en la producción y reducción de los costos directos e indirectos.

Continuando con el desarrollo, Aguerrondo concibe a la innovación como "acontecimientos concretos... que se generan, implementan y desarrollan" (1996: 23). Nuestro proyecto transita por las etapas descriptas por la autora, es decir: la gestación, la implementación y el desarrollo. En todas ellas ocurren procesos internos propios, cuyas características inciden sobre las etapas siguientes y que sobre todo pueden condicionar la viabilidad y permanencia de la innovación.

Relativo a la gestación, mencionaremos que los pre-requisitos desencadenantes están dados. Por un lado, tenemos la disfunción detectada (existe consciencia de que el actual dispositivo y su modalidad resultan insuficientes); por otro, está presente en la Unidad involucrada "la masa crítica de ideas" (Aguerrondo 1996: 24).

En lo estructural, factor condicionante de la oportunidad de inserción de la propuesta según la "coyuntura de poder de los grupos interesados" (Aguerrondo,1996: 24), diferenciamos varios niveles, la Gerencia de Recursos Humanos, el sector informático y las jerarquías máximas de las áreas de negocios. La primera, tiene conciencia sobre la insuficiencia del dispositivo, apoya y fomenta la propuesta; la segunda percibe a la experiencia como optimizadora de los recursos que administra, y la tercera presenta una muy buena disposición, sobre todo por contemplar una de sus preocupaciones, la baja del impacto en la producción operativa en tanto se ejecuta la capacitación. Encontramos entonces desde lo estructural, un escenario favorable.

La validación "del portador de la innovación" (Aguerrondo, 1996:24-25), esto es, el o los actores que son los responsables del emprendimiento, es esencial para que la propuesta pueda abrirse paso en la realidad (aspecto coyuntural). Aquí también encontramos las condiciones dadas, la unidad de capacitación es su eje central recibiendo el aporte del apoyo tecnológico brindado por la unidad informática.

Finalmente resta la elaboración de la propuesta de innovación, determinante de los subsiguientes procesos en las etapas posteriores. Respecto de la misma señalamos que el proyecto cumple con las características de no improvisado (presentado como proyecto corporativo alineado a los estándares organizacionales), y participativo (adoptando el modelo de participación diferencial).

Transitados los procesos anteriores el proyecto está en condiciones de ponerse en práctica, es decir, de pasar a su etapa de implementación. En ésta encuentra situaciones condicionantes que tienen que ver fundamentalmente con las resistencias que crea. La viabilización del mismo estará determinada por la forma en cómo se enfrenten y resuelven dichas resistencias y de cómo se inserte dentro del marco de las representaciones de los diferentes grupos que serán afectados. Su comprensión y la percepción de un beneficio y no de una amenaza, permite establecer las estrategias de viabilización a través de las capacidades de negociación, alianza y cooperación. 
En su implantación el emprendimiento requiere de recursos humanos y materiales para su ejecución. Un correcto dimensionamiento de éstos en función de los objetivos planteados es absolutamente necesario para la coherente formulación de nuestras metas y fundamentalmente para el logro de los resultados esperados. Es necesario la definición de un plazo temporal (cronograma) para la ejecución de actividades previstas y la presentación y evaluación de resultados esperados. La planificación de las diversas etapas y secuencias de actividades hacen a la eficiencia y eficacia con la cual vamos a llevar adelante nuestra empresa. Debemos tener muy en claro que si bien toda planificación debe ajustarse, los aplazamientos de etapas críticas pueden constituirse en eventos que comprometan la continuidad del proyecto. Finalmente corresponde mencionar el componente evaluación. Éste oficia como elemento de control de gestión durante el proceso de implantación y permite comprobar y ajustar el rumbo del proyecto, así como medir los resultados en términos de impacto.

En la etapa final, el desarrollo, el objetivo no puede ser otro que la consolidación de la propuesta, es decir, su afianzamiento y enriquecimiento, evitando la burocratización o la interrupción. Es indudable la necesidad de mostrar la consecución de los resultados buscados, así como también -y pensado desde el marketing -, generar en forma permanente la consciencia de valor, como lo señala García, "el producto... es un medio satisfactor, a través del cual el consumidor cumple con sus propósitos de satisfacción" (2001: 4).

\section{Aportes desde la didáctica}

Considerados los aportes desde la concepción de la innovación, continuaremos afirmando que en el ámbito organizacional el conocimiento aprendido es lo esencial a la hora de aplicar las competencias en el puesto de trabajo. Entendemos que sólo puede lograrse un aprendizaje de calidad si existe una propuesta de enseñanza de calidad, la cual tiene una identidad y características propias.

En este caso la experiencia implica un cambio profundo del escenario en donde tienen lugar los procesos de enseñanza y aprendizaje.

En primer lugar debemos considerar que las relaciones entre enseñante, aprendiz y saber son diferentes, no pudiéndose simplemente transferir las prácticas educativas realizadas tradicionalmente en el salón de clase. Ineludiblemente tenemos que pensar desde la didáctica, nuevas y eficaces formas de presentar los saberes.

Debemos concebir desde lo virtual, y en función de esto, cómo conducir el proceso de enseñanza determinando entre otras cosas:

- Cuáles son las configuraciones didácticas, es decir "la manera particular" en que el saber se "despliega para favorecer los procesos de construcción del conocimiento" (Litwin,1997: 97), adecuadas para fortalecer el proceso de aprendizaje en un público de adultos.

- Cómo operan los principios estructurantes para que el saber se presente como un todo coherente.

- Cómo se plantearán las funciones relativas al discurso del enseñante.

- La presencia del contrato didáctico y cómo formular las estrategias de ajuste del plan de enseñanza.

- Cómo establecer desde la transposición, la vigilancia epistemológica sobre las modificaciones del saber previendo mecanismos para evitar el desgaste biológico y moral.

- Qué características presenta la evaluación de las formas de intervención didáctica. 
En este camino nos han sido de particular valor las orientaciones aportadas por el artículo escrito por Lorenzo donde trata las diferencias entre los términos educación y enseñanza a distancia. De él extraemos referencias para "pensar modelos de intervención didáctica" (Lorenzo,2000: 6). En este trabajo se señala que "la enseñanza a distancia por su propia estructura y objetivos, brinda un ámbito de aprendizaje donde el adulto puede aprender aquello que personalmente le interese y responda a sus propias necesidades" (Pérez Serrano,1986: 3-7, citado por García Aretio, 1994:164, tomado de Lorenzo, 2000, op cit). Esta aseveración nos reafirma la adecuación de la generación de ámbitos de enseñanza no presenciales para un público adulto centrado en sus intereses y necesidades .

En referencia a la estructuración de una unidad y guía didáctica, Lorenzo cita a García Aretio, quien "...analiza y comenta la forma de organizar y dar secuencia a los contenidos..." (Lorenzo, op cit). García Aretio sostiene que "repeticiones, analogías, ejemplos y comparaciones ayudan a presentar las mismas ideas de forma diferente, ello siempre atendiendo a la preparación y experiencias previas del lector que podría rechazar el texto por monótono" (García Aretio en Lorenzo, op cit). Aquí podemos interpretar "un rasgo de la propuesta didáctica" (Lorenzo, op cit) orientadora de la construcción de enseñanzas en una modalidad a distancia.

Sin embargo, no encontramos en la actualidad explicitados los modelos didácticos de la enseñanza en contextos no presenciales, constituyendo un verdadero desafío a la hora de pensar nuestra propuesta. Con respecto a esta ausencia, adherimos a lo suscrito por Lorenzo, en el sentido de que es un campo que presenta enormes oportunidades de investigación que aporten a la "conformación de un referente teórico necesario" (Lorenzo, 2000: 7).

\section{Aportes desde el aprendizaje}

Transitados los aportes desde la innovación y la didáctica, creemos necesario a esta altura recordar que entendemos a la capacitación como la parte de educación de los adultos que se vincula con su vida de trabajo (Blake, 1987: 21).

En este sentido no podemos obviar los aportes desde el aprendizaje contextualizado en un público adulto, realizado en un ambiente laboral pero además producido a través de una modalidad no presencial.

Volvemos a citar a Oscar Blake, consultor en capacitación laboral, quien señala que el adulto es un ser que valora su pasado el cual es fuente de su seguridad y "... no permitirá que se ignore o desvalorice su experiencia, no admitirá no ser escuchado y no tolerará metodologías que no lo involucren"(1987: 20).

Las propuestas de enseñanza para adultos dentro de una organización deben considerar estos elementos y crear un espacio que ubique al contenido de la capacitación como útil para el trabajo cotidiano. El participante debe poder visualizar el por qué y el para qué se lo capacita como requisito indispensable para generar condiciones favorables para la enseñanza y el aprendizaje. Requiere en suma, "la generación de genuinos y significativos ambientes de aprendizaje" (Lorenzo,2000: 6).

En la realidad organizacional descripta (dinámica, exigente, en proceso de cambio), cada persona necesita desarrollar la motivación, su autoestima y las herramientas que le permitan encontrar y alcanzar todas sus potencialidades. Ello será posible por el reconocimiento de la importancia del aprendizaje metacognitivo: es decir, la habilidad de los aprendices en monitorear y controlar sus propios procesos de aprendizaje.

Tal como plantea McCombs (1992), citado por Fainholc (1999: 5) respecto de cómo se da el proceso de aprendizaje en el estudiante, sostiene que éste:

- Opera holísticamente a través de sus de características intelectuales, emocionales, sociales, culturales y físicas. 
- Se desarrolla a través de todos los dominios de sus competencias, nunca es estático, y presenta un proceso de crecimiento dinámico que abastece a las necesidades inherentes para lograr el dominio, su control y la pertenencia.

- Basa el comportamiento y el desarrollo de sus capacidades en sus percepciones, en el procesamiento socio-cognitivo-cultural y en las evaluaciones de las situaciones que realiza, construyendo su conocimiento a partir de lo que evalúa como relevante según sus metas e intereses personales y grupales.

Desde esta perspectiva consideramos que la propuesta de innovación -en relación a la consideración del aprendizaje de los participantes-, debe configurarse desde los aportes de las teorías cognitivas (el constructivismo piagetiano, las teorías contextualistas o socio-históricas, la teoría del aprendizaje significativo), las cuales intentan explicar el mecanismo por el cual los sujetos aprenden y modifican sus propios sistemas conceptuales de interpretación.

Esto nos lleva a encarar a los estudiantes como personas que poseen formas diversificadas de representación mental y lenguajes intelectuales. En consecuencia debemos poner el acento en la forma en que cada estudiante tiene de apropiarse y construir conocimiento, valorizando el autoaprendizaje y considerándolo como autor de su propio desarrollo cognitivo.

Tomando en cuenta el paradigma de la teoría cognitiva, caracterizaremos y consideraremos al estudiante de la siguiente forma:

- Activo.

- Atribuye significados.

- Tiene una forma de organización interna.

- No es un organismo vacío ilimitadamente moldeable.

- El aprendizaje requiere significatividad.

Esta concepción transformará los programas y diseño de cursos organizacionales en una propuesta de educación a distancia, donde las situaciones de enseñanza en contextos virtuales apoyen la mejora y el acrecentamiento en los estudiantes de las estrategias de aprendizaje autónomo.

\section{Aportes desde la concepción de educación a distancia}

Nuestra propuesta, donde "las distintas formas de estudio a todos los niveles no se encuentran bajo la continua, inmediata supervisión de los tutores" (Garcia Arieto en Lorenzo 2000: 5), requiere necesariamente del aporte de la concepción de educación a distancia.

A tales efectos consideramos necesario revisar algunas de sus conceptualizaciones clásicas con el propósito de identificar sus características para poder iluminar el proceso de implantación.

Moore expresa que la educación a distancia, "consiste en todos los arreglos para proveer educación a través de medios de comunicación... a personas vinculadas a aprendizajes planeados en lugares y tiempos diferentes al de los instructores" (Moore, 1990 en Fainholc 1999: 23-25).

Escotet indica que la misma "consiste en una educación que se entrega a través de un conjunto de medios didácticos que permiten prescindir de la asistencia a clases regulares y en la que el individuo se responsabiliza por su propio aprendizaje" (Escotet, 1980 en Fainholc op cit).

Holmberg señala que "son las diferentes formas de estudios a todos los niveles que no se hallen en continua e inmediata comunicación entre profesores y estudiantes,... sin embargo se benefician de la planificación, guía y evaluación en una organización de tutoría" (Holmberg, 1977 en Fainholc op cit). 
Rowntree establece "es el sistema de enseñanza en el que el estudiante realiza la mayor parte de su aprendizaje por medio de materiales didácticos, con un escaso contacto directo con los profesores" (Rowntree, 1986 en Fainholc op cit).

La propuesta de Keegan (citado por Lugo y Schulman,1999: 11), entiende al proceso de educación a distancia como: "...la cuasi permanente separación de profesores y estudiantes a lo largo de la mayor parte del proceso de aprendizaje". En éste se acude a medios técnicos para: establecer la relación entre el profesor y estudiante, procurar una comunicación bidireccional y oficiar de soporte de los contenidos del curso. La cuasi permanente ausencia del aprendizaje en grupo es una de las características fundamentales que se da lo largo de la mayor parte del proceso de aprendizaje.

De lo expresado por los diferentes autores podemos caracterizar a la educación a distancia como el proceso educativo definido por: un acceso según el "autotempo" y responsabilidad de los estudiantes, basado en diversas tecnologías como fuentes de información y soporte de contenido, los cuales no son presentados necesariamente en forma lineal sino como situaciones de comunicación "empática, para alcanzar -a través de la enseñanza guiada-, la construcción del saber y el aprendizaje" (Fainholc, 1999: 26)

\section{Aportes desde la tecnología educativa}

Hasta ahora en nuestro marco conceptual nos han auxiliado la innovación, concepciones de enseñanza y aprendizaje y la educación a distancia. Sin embargo necesitamos -pensados desde la virtualidad- medios y herramientas distintas. Consideramos entonces necesario también el aporte que nos brinda la Tecnología Educativa.

Litwin señala a la tecnología educativa como "... el cuerpo de conocimientos que, basándose en disciplinas referidas a las prácticas de enseñanza, incorpora todos los medios a su alcance y responde a la consecución de fines en los contextos sociohistóricos que le otorgan significación" (Litwin y otros, 1995 : 26), agrega Maggio "la tecnología educativa, al igual que la didáctica, se preocupa por las prácticas de la enseñanza, pero a diferencia de ésta incluye entre sus preocupaciones el análisis de la teoría de la comunicación, y de los nuevos desarrollos tecnológicos: la informática en primer lugar..." (Maggio, en Litwin y otros, 1995: 27).

Tal como se expresó en el marco de la capacitación virtual, docente y estudiante están separados por la distancia o por distancia y tiempo, dependiendo la misma de la tecnología como mediador en el proceso de la comunicación.

Podemos señalar que la tecnología en este proyecto se basa en herramientas destinadas a la creación de materiales multimedia, editores de páginas Web, software de comunicación y las diseñadas específicamente para la distribución y evaluación de cursos a través de la Intranet. Actualmente existe a disposición gran cantidad de productos para la creación de entornos de enseñanza-aprendizaje, tantos que, quizás como afirman, McGreal, Gram y Marks "el problema sea determinar qué herramientas serán más adecuadas para lograr unos objetivos educativos específicos" (McGreal, Gram y Marks en De Benito, 2000: 1).

Para ello, debemos determinar, por una parte, cuáles son las necesidades y, por otra, cuáles las posibilidades de las herramientas de que disponemos. La viabilidad del proyecto depende de la adecuación y adaptación de las tecnologías a la realidad organizacional.

En este marco -como se indicó-, docente y estudiante dependen de la tecnología como mediador en el proceso de la comunicación y como señala Adell éstas "han desmaterializado, deslocalizado y globalizado la información" (1997: 11), eliminando la necesidad de los participantes de una actividad, de coincidir en el espacio y en el tiempo. 
En este punto es necesario precisar que la experiencia se funda, tal como se expresó en el propósito, en la utilización de aplicativos en ambiente Intranet. Esta opción se identifica como la más adecuada en virtud del actual desarrollo tecnológico de la organización, de la magnitud de su red informática, así como de su incorporación a la cultura organizacional.

Esta selección nos ubica en lo que Lugo y Schulman, categorizan como "tercera etapa" (1999: 17) en la evolución de la educación a distancia. En ella las nuevas tecnologías de la información y comunicación, es decir, el "conjunto de procesos y productos derivados de las nuevas herramientas (hardware y software), tales como los soportes de la información, canales de comunicación...., procesamiento y transmisión digitalizados de la información" (González y otros, 1996 en Lugo y Schulman, 1999: 18) y "su nuevo paradigma, las redes informáticas" (Lugo y Schulman, op cit) proporcionan una amplia gama de herramientas por las cuales se mediatiza el uso de la función simbólica y en consecuencia proveerán el ambiente de enseñanza - aprendizaje a través de la concreción de:

- Creación, organización, distribución y recepción de contenidos.

- Interacción con otros ordenadores en un proceso de simulación de comunicación directa o indirecta entre personas.

- Sistemas de evaluación del índice de satisfacción y aprendizaje de los participantes.

En lo concreto, este ambiente permitirá la utilización de soportes y herramientas tales como los tutoriales, bases de datos en línea, bibliotecas electrónicas, hipertextos distribuidos, simulaciones de procesos, representaciones gráficas, integración de texto, imagen y sonido, navegación hipertextual, etc.

Creemos importante mencionar -suscintamente- el impacto que innovaciones similares han tenido en diferentes empresas. Con ello trataremos de descubrir cómo puede beneficiar a la organización el análisis de experiencias exitosas.

Brandon Hall (2001: 85), uno de los máximos especialistas de la industria del aprendizaje electrónico, nos aporta varios ejemplos relevantes de reconocidas empresas mundiales. Entre ellos encontramos a Price Waterhouse que capacitó virtualmente en el área de auditoría a 7000 profesionales en más de 50 países, Intel Corp. a través de la instrucción basada en medios informáticos a más de 800 empleados, IBM ha implementado en modalidad de capacitación virtual su programa de formación gerencial con la intervención de más de 3500 nuevos gerentes.

Sería impensable trasladar directamente la experiencia de estas multinacionales a una organización pública uruguaya. Resulta evidente que existen diferencias en roles y objetivos entre estas compañías y la institución donde esta innovación se lleva a cabo. El lucro es el eje central de la gestión de una empresa multinacional, lo cual dista de ser el caso de los fines sociales de la institución estatal. Estas diferencias están -inevitablemente- reflejadas en la gestión de los Recursos Humanos y sus respectivas áreas de formación. Debemos apreciar que en los casos citados se gira en torno al entrenamiento corporativo ("corporate training"), en tanto la realidad de la organización apunta hacia la formación funcional referida al servicio. Esta diferencia es significativa en términos de misión y visión de los actores, asignación de recursos y motivos de "generación" de la innovación.

De todos modos resulta relevante que la experiencia en el mundo de las grandes corporaciones señale inequívocamente (según las propias corporaciones) que la capacitación distribuida genera indicadores sustancialmente mejores a los obtenidos mediante las metodologías tradicionales a un costo sensiblemente inferior. 


\section{Evaluación de la propuesta: componente investigación}

Nuestro proyecto tiene como finalidad una innovación, pero la sustentabilidad de progresos en el área de formación requiere "del rigor de la evaluación" (Le Boterf,1993: 71). Se establece entonces la necesidad de una evaluación de la innovación a nivel organizacional.

Considerando que en la Organización el conocimiento aprendido es lo esencial a la hora de aplicar las competencias en el puesto de trabajo y considerando también la intención de que este aprendizaje se genere a través de una nueva modalidad, nos planteamos las siguientes interrogantes:

- Al finalizar el ciclo de formación, ¿se encontrarán presentes las competencias en el puesto de trabajo de similar forma, (cantidad y calidad), a que si el acto formativo se hubiese efectuado a través de modalidades presenciales?

- ¿Cómo perciben los participantes el cambio de modalidad?, ¿se sintieron afectados por el mismo (negativa o positivamente)?, ¿qué influencia , respecto del aprendizaje, tienen las representaciones que éstos se forman de cómo aprendieron?

- Los distintos aspectos que involucran el acto de enseñanza, ¿fueron facilitadores o inhibidores a la hora de incorporar y posteriormente transferir los conocimientos en el trabajo?.

En suma: ¿los participantes de los programas de formación aprenden y transfieren con igual eficacia independientemente de la modalidad?

Esto nos lleva a la necesidad adicional en la propuesta de innovación, de plantearnos la evaluación de la experiencia formulando un componente de investigación, el cual consideramos debe basarse en dos dimensiones:

- La evaluación de la percepción de los participantes frente al cambio de modalidad a través de una investigación de corte cualitativo.

- La evaluación del aprendizaje sobre la base de un análisis comparado entre las dos modalidades de formación, adoptando el modelo de corte cuantitativo.

Es nuestra intención dejar al menos planteado este componente de investigación y su pertinencia en el proyecto.

\section{Metodología}

\section{De Innovación}

En lo concreto, la innovación se plasma en la formulación de un proyecto, es así que adoptamos como metodología la propia de desarrollo de proyectos, alineada con la normativa institucional de la organización donde se impulsa la propuesta.

A efectos de no recargar la redacción no se abundará en información detallada sobre la misma, mencionando solo que su estructuración requiere la definición de objetivos, actividades, cronograma e indicadores.

Para que el lector pueda formar una opinión sobre los contenidos concretos de la propuesta de innovación y su alineación con el marco conceptual, expondremos del mencionado proyecto su objetivo general y los resultados esperados.

El objetivo general es implementar y reglamentar un sistema de capacitación a distancia en competencias laborales, con procesos de gestión y evaluación, basado en la utilización de aplicativos en ambiente Intranet. 


\section{Los resultados esperados son:}

- Implementar cursos en modalidad virtual utilizando las herramientas adecuadas a la Intranet corporativa.

- Implementar un sistema de evaluación sobre un soporte informático que permita la recolección, registro, corrección y devolución de resultados de aprendizaje. Incluimos en este ítem la evaluación del grado de satisfacción del alumno sobre los distintos componentes de los procesos de enseñanza y aprendizaje.

- Diseñar un sistema de control, monitoreo, tutoría y seguimiento de actividades de alumnos estableciendo los criterios y herramientas que provean la debida orientación y la generación de interacciones significativas.

- Generar un sistema de perfiles de acceso, a niveles que permita la regulación del acceso a los distintos niveles de un mismo curso o a diferentes cursos

- Elaborar un sistema gestor que soporte esta modalidad optimizando y simplificando la gestión de los actores, registro de la intervención y acciones de capacitación.

- Producir un reglamento sobre las obligaciones y derechos de los actores que establezca un marco normativo con el fin de brindar la validez y transparencia necesaria.

\section{De Investigación}

Como se expresó, nuestro proyecto tiene como centro el desarrollo de una propuesta de innovación, sin embargo es nuestra intención dejar al menos planteado el componente de investigación requerido por la evaluación de la experiencia. Implementar esta investigación plantea varios retos complejos.

Por un lado la selección y desarrollo en ambiente virtual de un curso que tenga la complejidad conceptual necesaria para validar la experiencia. Éste a su vez, debe estar dictándose en la modalidad presencial y tener establecidos los parámetros de evaluación estandarizados para que los resultados puedan ser comparables.

Por otro, se nos plantea la dificultad de la conformación de dos grupos de estudio equivalentes, controlando distintas variables que pueden afectar el resultado (edad, diversidad de base de formación, área de negocio, etc.).

El sentido de oportunidad no es de menor peso. Con esta expresión queremos señalar lo estratégico de la selección del momento en que se dispare la investigación. Como hemos indicado anteriormente, la sola aparición de una nueva forma de hacer las cosas ocasiona resistencias. Entendemos que una efectiva evaluación requiere cierta familiarización organizacional previa con la experiencia en cursos de esta modalidad. De lo contrario -estimamos- correríamos el riesgo de recoger datos sesgados por la aparición de la innovación, que no estarían midiendo exactamente lo que queremos: la comparación entre ambas modalidades.

Brevemente indicaremos que la experiencia consistiría en la formación de dos grupos equivalentes a los cuales se les impartiría el curso identificado en distinta modalidad (Grupo A- presencial, Grupo Bvirtual). Cada grupo estaría constituido por un mínimo de 15 y un máximo de 20 personas.

Culminado el mismo se compararán, de acuerdo con la pauta de evaluación existente, los resultados entre los distintos trabajos, intentando identificar diferencias en las diversas variables medidas.

Respecto a la investigación sobre la percepción de los participantes en cuanto a la incidencia de la modalidad en los aprendizajes, la misma tendría un corte cualitativo, basándose esencialmente en la entrevista a los participantes. 
Su carácter sería exploratorio y descriptivo intentando hallar y describir en la percepción de los participantes los factores que incidieron favorable o negativamente en el proceso de adquisición de conocimientos.

Nuestra intención es concretar este componente de investigación una vez que la innovación se encuentre implementada y consolidada.

\section{La implementación}

Como se expresó anteriormente, ha sido aspiración de este trabajo convertirse en una realidad concreta dentro de la organización para la cual fue pensada. Este camino se ha ido recorriendo e intentaremos tangibilizar la experiencia para el lector presentando los productos entregados e implementados hasta la fecha.

A tales efectos, comenzaremos señalando que se han generado beneficios «adicionales» a los previstos por la propuesta. Ello para indicar que la experiencia nos deja con la convicción de que si bien la innovación debe ser planificada, también debe gestionarse con amplio criterio de flexibilidad. Éste nos permite identificar la posibilidad de adicionar productos no previstos, que con poco esfuerzo o costo adicional, representan un aumento del valor agregado en el resultado de la innovación. También nos permite capitalizar circunstancias que nos hacen avanzar con mayor eficacia en la propuesta sorteando limitantes existentes.

Como consecuencia se generaron productos no concebidos originalmente, tales como el portal de toda la Gerencia de Recursos Humanos, agregándole presencia institucional a tres unidades más. Se desarrolló puntualmente el sitio de Capacitación, el cual presenta información general, cronograma de cursos, ofertas internas y externas, evaluación, foros, así como se dispararon propuestas para generar líneas de trabajo sobre gestión del conocimiento.

Respecto de los productos concebidos originalmente e implementados, los hemos dividido en las siguientes categorías referentes al diseño e implementación de:

- cursos en modalidad web.

- sistema de evaluación on line de resultados de aprendizaje.

- sistema de tutoría.

- sistema informático de accesos y registro de autorizaciones según perfiles.

- sistema de evaluación de satisfacción del participante.

\section{Cursos en modalidad web}

Dentro del sitio de Capacitación se creó la opción "cursos on line". En la misma, se ha dispuesto la información dispersa en Intranet generada por la unidad de capacitación a la cual se le ha dado el formato de cursos. Se trata fundamentalmente de contenidos sobre nuevas aplicaciones de utilización masiva o que forman parte de información de difusión permanente. Este avance posiciona a los usuarios frente a la nueva modalidad y predispone la cultura organizacional al cambio que se está implementando.

Un primer curso se trató de la capacitación de un aplicativo corporativo destinado a dos niveles de funcionarios: operativos y de supervisión. El curso completo consta de 13 lecciones divididas en dos módulos; su estructura no es lineal, pudiendo el participante navegar libremente por los contenidos según su centro de interés. Se estableció una guía de estudio para que el participante comprenda el nuevo ambiente de enseñanza que se le presenta y cómo enfrentarlo. Adicionalmente, se proponen ejercicios de autoevaluación y ejercitación en el ambiente real de trabajo. Se desarrollan las preguntas claves, es decir aquellos casos que recurrentemente se presentan en la operativa, permitiendo a los usuarios acceder a la lección - o fracción de lección- según las dudas específicas (capacitación "just in time"). 
La interacción con docentes se realizó telefónicamente, hacia una unidad de "mesa de ayuda" donde se resolvieron las diversas consultas.

Adicionalmente, en una segunda etapa, se transformó material de otros tópicos. A diferencia del carácter estático planteado en el anterior desarrollo, se incorporó la dinamización de ciertos contenidos.

Continuando con el desarrollo de nuestro proyecto, nos encontramos dictando virtualmente un curso de actualización destinado a los instructores de la Unidad con el objetivo de proporcionarles un marco teórico e instrumental para la formulación y aplicación de pruebas de evaluación.

Es relevante mencionar que en este curso por primera vez se utilizaron:

- el sistema de acceso restringido.

- el sistema de evaluación on line.

- el foro, mediante el cual se ejecutó parte de la tutoría.

Si bien es muy temprano para sacar conclusiones se puede hacer mención a algunas observaciones y algunos resultados obtenidos.

- En el curso donde se controló la variable asistencia (evaluación docentes), no existió abandono.

- Las pruebas efectuadas por el sistema de evaluación on line arrojaron una calificación superior al 80\%.Dada la complejidad del material indica un buen nivel de autoestudio y de adhesión a la propuesta.

- En general la necesidad de consultas se redujo drásticamente hacia la mitad del curso; algunos participantes expresaron en sus comunicaciones que se debió a una baja en la ansiedad al ir transitando por la nueva situación.

- Los comentarios finales han sido favorables y se expresó casi por la totalidad de los participantes, la "ventaja" de disponer del curso a "toda hora" en forma independiente a la situación de clase.

También es interesante hacer mención a la adaptación de los docentes que dictaban el curso en la nueva modalidad. En un primer momento se manifestaban actitudes de inseguridad en las interacciones y escepticismo sobre los resultados que se obtendrían. Estas inseguridades se fueron mitigando a medida que transcurría el curso; concretamente hacia el fin del primer módulo la actitud y comentarios mencionados cambiaron de forma notoria, observándose una buena integración y cambio de opinión hacia la modalidad.

\section{Sistema de evaluación on line de resultados de aprendizaje}

Uno de los objetivos de nuestro proyecto plantea la formulación de un sistema de evaluación en modalidad virtual, esto es, el alumno evaluado en el mismo ambiente donde aprende.

Corresponde aclarar que la instancia de evaluación presencial no es excluyente y dependerá de la decisión técnica que se tome para cada curso. Sin embargo, dentro de nuestro proyecto nos planteamos la necesidad de diseñar un sistema que por sí mismo realizara evaluaciones Éste debería ser capaz de desplegar los contenidos ante el usuario, garantizar la integridad de las pruebas, resolver la corrección y finalmente, comunicar el resultado al participante.

Se optó por el desarrollo interno de una aplicación. El sistema se encuentra actualmente finalizado y en utilización. Funciona a través de preguntas de múltiple opción, desplegándole al participante las seleccionadas randómicamente por parte del programa. A su vez se incorporó el parámetro tiempo, dándole al usuario un tiempo limitado para la realización de la prueba.

La corrección es automática: el proceso compara las respuestas efectuadas contra los parámetros validados como correctos dentro de las preguntas y el nivel mínimo porcentual de aprobación; el resultado es informado al usuario en tiempo real y se almacena en una base de datos, generándose una historia que el propio funcionario o los administradores pueden consultar. 


\section{Sistema de tutoría}

Se definió la utilización de tres herramientas a los efectos de formular un sistema de tutoría a distancia. La opción por una o la utilización combinada de ellas se determinará según la naturaleza del curso y el diseño técnico del mismo.

La más básica de éstas, el teléfono, crea espacios de interacción verbal con los estudiantes.

En el ambiente informático se opta por la utilización del correo electrónico o el foro, elemento específicamente desarrollado para esta innovación.

El foro tradicional permite el despliegue de los mensajes anidados según un tema en particular, facilitando el ordenamiento del intercambio de la comunicación. El docente oficia como disparador de las distintos "mensajes nuevos" que inician la categorización de otra dimensión en la discusión.

El registro de datos se almacena en una base de datos lo cual posibilita un análisis de los comentarios registrados, pudiendo a través del mismo formular las mejoras necesarias en el curso y generar una base de datos de conocimientos con las preguntas y respuestas más relevantes, importantes o frecuentes.

\section{Sistema informático de accesos y registro de autorizaciones según perfiles.}

Tanto el acceso a los cursos como las evaluaciones requerían implementar un sistema que habilitara o restringiera el acceso de los usuarios. Se diseñó un aplicativo que permite el control de los mismos. El nivel de administración habilita a los participantes según su usuario de red.

\section{Sistema de evaluación de satisfacción del participante.}

La evaluación de la satisfacción del participante se solicita cada vez que un participante finaliza un curso. Ésta se resolvió estableciendo bases de datos en línea donde el usuario responde a la encuesta propuesta. La información guardada permite la emisión de varios reportes de satisfacción categorizados según las dimensiones que agrupan las distintas variables.

\section{Algunas reflexiones finales}

Como señaláramos al inicio de nuestro trabajo tomamos el reto de "desaprender" para volver a aprender, a través de una experiencia de innovación que tenemos la fortuna de poder concretar y no de dejarla a nivel de expresión de deseo.

Ésta incorporó un significativo cambio en las estrategias en el área de capacitación de un importante organismo estatal: la implantación de un sistema de capacitación virtual.

Este emprendimiento ha afectado los ejes fundantes del área y abierto una cantidad considerable de nuevas oportunidades para la optimización de la capacitación y de la formación de los recursos humanos de la institución.

No pocas fueron las dificultades en la ejecución de la propuesta. Tal como reseñábamos en nuestra introducción, hacer las cosas de otro modo implica de por sí resistencia al cambio; sin embargo, terminamos con la convicción de que la fuerza de argumentos valederos, junto con la pertinencia de la propuesta, empuje y presencia de productos concretos, prevalecen.

Al momento de la redacción de este artículo se diseñó un curso sobre un aplicativo concreto con un público objetivo cercano a los 4.000 funcionarios; una instancia de difusión de conceptos de seguridad corporativa para otros 2.000 funcionarios, y un curso de actualización del cuerpo docente interno. Junto a esto se han diseñado y se encuentran en funcionamiento, aplicativos de evaluación, control y tutoría. 
Si bien es temprano para extraer conclusiones, se ha percibido a nivel general que la modalidad ha sido bien recibida y aceptada como «cosa buena» por la cultura organizacional.

Nos resta continuar trabajando para consolidar el emprendimiento y desatar las líneas de investigación expresadas intentando capitalizar las enseñanzas que esta experiencia nos brinda.

\section{Bibliografía}

Adell, J. 1997. Tendencias en educación en la sociedad de las tecnologías de la información. En Rev. Edutec No. 7/ noviembre 1997.

Aguerrondo, I. 1996. Innovación y Calidad de la Educación. En: Rev. Lationoamericana de Innovación Educativa, Buenos Aires: OEA.

Blake, O. 1987. La capacitación : Un recurso dinamizador de las organizaciones. Buenos Aires: Epso

De Benito, B. 2000. Herramientas para la creación, distribución y gestión de cursos a través de Internet. En Rev. Edutec, No. 12/junio 2000.

Fainholc, B. 1999. La interactividad en la educación a distancia. Buenos Aires: Paidós.

Fainholc, B. 1999. Algunas consideraciones sociopedagógicas y tecnológicas para las acciones tutoriales en entornos de educación virtual. Buenos Aires: Paidós.

García, J. C. 2001. Marketing aplicado a los Servicios Públicos. Montevideo: Banco de Previsión Social.

Le Boterf, G. y Otros 1993. Cómo gestionar la calidad de la formación. España: Aedipe.

Hall, B. 2001. Revista Gestión Volumen 6 set-oct 2001.

Litwin, E. y Otros 1995. Tecnología Educativa: Política, historias, propuestas. Buenos Aires: Paidós.

Litwin, E. 1997. Las configuraciones didácticas. Buenos Aires: Paidós

Lorenzo, E. 2000. Enseñanza a distancia y educación a distancia: ¿de qué estamos hablando? Cuadernos de Investigación Educativa Vol 1 No.6 julio 2000. Montevideo: Universidad ORT.

Lugo, T. y Schulman, D. 1999. Capacitación a distancia: acercar la lejanía. Buenos.Aires: Magisterio.

* Diploma en Educación, Universidad ORT. Profesor de Idioma Español, Instituto de Profesores Artigas. Maestro. 